\section{(2) OPEN ACCESS}

\title{
Palliative and end-of-life care and
}

\section{junior doctors': a systematic review and narrative synthesis}

\section{Aamena Bharmal (D) , ${ }^{1,2}$ Tessa Morgan, ${ }^{1}$ Isla Kuhn, ${ }^{3}$ Bee Wee, ${ }^{4,5}$ Stephen Barclay}

\section{ABSTRACT}

published online only. To view please visit the journal online (http://dx.doi.org/10.1136/ bmjspcare-2019-001954).

${ }^{1}$ Primary Care Unit, University of Cambridge, Cambridge, UK ${ }^{2}$ Cambridge University Hospitals NHS Foundation Trust, Cambridge, UK

${ }^{3}$ Medical Library, University of Cambridge, Cambridge, UK

${ }^{4}$ Sir Michael Sobell House Hospice, Oxford, UK

${ }^{5}$ University of Oxford Harris Manchester College, Oxford, UK

\section{Correspondence to} Dr Aamena Bharmal, University of Cambridge Primary Care Unit, Cambridge CB2 OSR, UK; aamena.bharmal@gmail.com

Received 4 July 2019 Revised 9 October 2019 Accepted 28 October 2019 this care.

\section{(D) Check for updates}

(c) Author(s) (or their employer(s)) 2019. Re-use permitted under CC BY-NC. No commercial re-use. See rights and permissions. Published by BMJ.

To cite: Bharmal A, Morgan T, Kuhn I, et al. BMJ Supportive \& Palliative Care Epub ahead of print: [please include Day Month Year]. doi:10.1136/ bmjspcare-2019-001954
Background Palliative and end-of-life care is a core competency for doctors and is increasingly recognised as a key clinical skill for junior doctors. There is a growing international movement to embed palliative care education in medical student and junior doctor education. To date there has been no review of the literature concerning the views and experiences of junior doctors delivering

Aim To review the published literature between 2000 and 2019 concerning junior doctors' experience of palliative and end-of-life care. Methods Systematic literature review and narrative synthesis.

Results A search of six databases identified 7191 titles; 34 papers met the inclusion criteria, with a further 5 identified from reference searching. Data were extracted into a review-specific extraction sheet and a narrative synthesis undertaken. Three key themes were identified: (1) 'Significance of death and dying': all papers found that junior doctors care for many patients approaching the end of life, and this often causes emotional distress and can leave persisting memories for many years afterwards; (2) 'Thrown in at the deep end': junior doctors feel unprepared and unsupported in providing palliative and end-of-life care; and (3) 'Addressing the gaps': junior doctors often experience a medical culture of disengagement towards dying patients and varying attitudes of senior doctors. Subsequently they have to learn the skills needed through seeking their own opportunities.

Conclusion Medical education needs to change in order to better prepare and support junior doctors for their role in caring for dying patients. This education needs to focus on their knowledge, skills and attitudes.

\section{INTRODUCTION}

Acute hospital wards are the most prevalent place of death across the world: $47.4 \%$ of deaths in England and Wales in $2016^{1}$ and 58\% globally. ${ }^{2}$ Most hospital palliative and end-of-life care is provided by patients' normal clinical teams rather than palliative care specialists, ${ }^{3}$ where junior doctors mainly work and are at the front line of end-of-life care provision. ${ }^{4}$

Palliative and end-of-life care has therefore been recently described as 'a core competency' for all doctors because of the need to have excellent skills in clinical assessment, communication, multidisciplinary teamwork and prescribing to provide this care for patients. ${ }^{5}$ Across the globe there is an increasing emphasis on embedding palliative care in the education of all medical students and doctors. ${ }^{5}$ However the literature suggests that medical student education in this subject area is patchy and frequently inadequate. ${ }^{7}$ There is limited understanding of how this affects recent graduates' provision and experience of palliative and end-of-life care.

We therefore undertook a systematic review of the literature concerning junior doctors' experience and views of providing palliative and end-of-life care. To our knowledge no such review has been previously undertaken.

\section{Aim}

The aim was to undertake a systematic review and narrative synthesis of the international literature concerning junior doctors and end-of-life care focusing on the following:

- What is their experience of providing care?

- What are their attitudes towards providing care?

- How adequately prepared do they feel?

- How supported do they feel?

\section{METHODS}

\section{Search strategy}

A search strategy was devised in collaboration with a professional medical librarian 
((exp Palliative Care) or (palliat $\left.{ }^{\star}\right)$ or (exp Death/ or death) or (exp Terminal Care/ or "end of life") or (terminal* adj3 (care or ill*) or (dying) or (dnr or "do not resuscitate" or dnar) or (resuscitat*) or (Resuscitation/ or exp Resuscitation Orders) or (exp

Advance Directives/ or exp Withholding Treatment).ti,ab.

AND

((junior* or trainee* or training) adj2 (doctor* or physician* or clinician*)) or (exp "Internship and Residency") or (fy1 or fy2) or (foundation adj year*) or "house officer*".ti,ab. (1824)

(sho or cmt or cst or rmo).ti,ab.

AND

((perception*) or (perceiv*) or (experienc ${ }^{*}$ ) or (view* or opinion*) or (coping) or (exp Attitude/ or exp "Attitude of Health Personnel"/ or exp Attitude to Death/ or attitud*) or (exp Attitude to Health) or (train or trained) or (confidence) or (need) or (exp Health Knowledge, Attitudes, Practice/) or (exp Education Department, Hospital/ or exp Education, Medical/ or exp Education, Professional, Retraining/ or exp Education, Graduate/) or (exp Learning/ or learn*) or (cope) or strateg*) or (support*) or (exp Inservice Training/) or (exp Interview/ or interview*) or (exp "Surveys and Questionnaires"/) or (exp Clinical Competence/ or exp Professional Competence/) (competenc $\left.{ }^{\star}\right) \cdot \mathrm{ti}, \mathrm{ab}$

Figure 1 Medline search strategy.

(IK). An initial scoping search strategy was reviewed against the inclusion and exclusion criteria, following which the definitive search strategy was developed (figure 1).

The inclusion and exclusion criteria are summarised in figure 2. Due to a review published in 2000 demonstrating widespread palliative and end-of-life care education in medical undergraduate curricula, we sought to review the effect of this undergraduate teaching on junior doctors' experience. ${ }^{8}$ For the purpose of the review, we define 'junior doctors' as those who had graduated from medical school but had not yet entered a specialty training programme; for example in the UK, this would be the foundation programme, core medical or core surgical training years (previously known as junior and senior house officers). Junior doctors predominately work in adult inpatient settings during the early years of their careers; therefore, only hospital or inpatient hospice settings were included. Care of children and young people under 18 years of age as well as bereavement care were excluded as they are considered specialist areas.

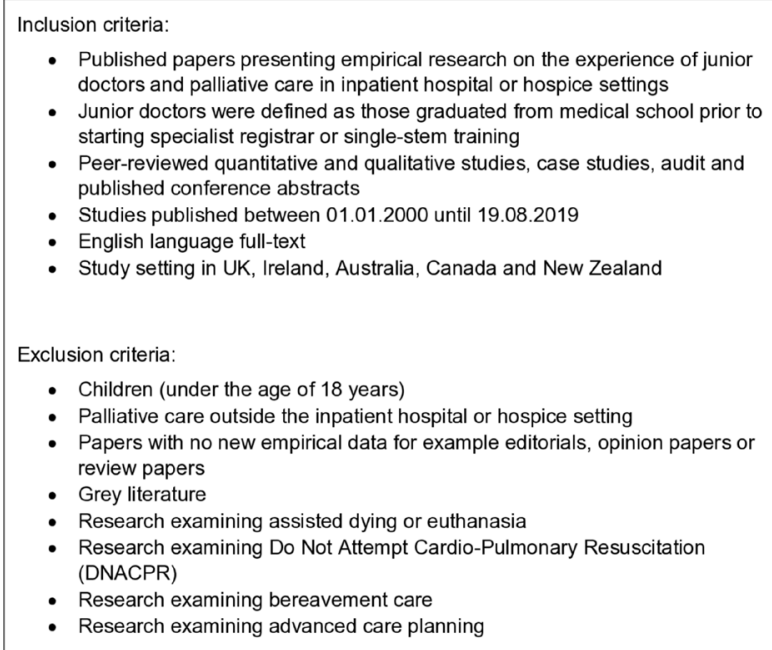

Figure 2 Inclusion and exclusion criteria.
The literature was restricted to countries with broadly similar healthcare services, medical training programmes and cultural contexts: the USA was excluded as doctors enter specialty training very early in their careers. Opinion pieces, literature reviews and editorials were excluded unless they contained original empirical data.

\section{Conducting the search}

We searched six electronic databases (Medline, Embase, PsycINFO, Web of Science, Scopus and Cochrane) for papers published between January 2000 and August 2019. The search was initially run until January 2018 and then subsequently updated in August 2019 to ensure that any additional papers were captured within this review.

The search results were downloaded into EndNote and duplicates were removed. All titles, abstracts and full-text papers were screened by the first reviewer $(\mathrm{AB})$, with a sample of abstracts and full-text papers screened by the second reviewer (TM). Any disagreements were resolved by review team discussion (AB, $\mathrm{TM}$ and $\mathrm{SB}$ ).

\section{Data analysis}

A narrative thematic analysis was used to synthesise the heterogeneous literature, which enabled an empirical 'data-driven' approach to identify themes.' Included papers were weighted by $\mathrm{AB}$, with a sample independently weighted by TM, for their contribution towards answering the review questions, using the Gough's weight of evidence framework, which measures each paper against rigour of study design, appropriateness to the review question and relevance to answering the question. ${ }^{10}$

\section{RESULTS}

Search results are summarised in the adapted Preferred Reporting Items for Systematic Reviews and MetaAnalyses flow chart (figure 3). Thirty-four papers were included from the database search, with a further five from reference searching of included papers. Of these 39 papers, 23 were from the UK and 7 from Canada, with 9 from across the globe; 17 used quantitative methods, 18 qualitative methods and 4 mixed methods. The quality of the papers varied on the Gough's weight of evidence: 11 weighted high, 16 medium and 12 low quality ( 7 of which were conference abstracts). The included papers are summarised in online supplementary file 1.

All the papers provided evidence that the majority of junior doctors provide palliative and end-of-life care for many patients early in their careers. ${ }^{11-49}$ An average of $40 \%$ (range: $36 \%-46 \%$ ) of junior doctors cared for 1-10 patients, while $51 \%$ (range: $40 \%-61 \%$ ) of junior doctors cared for more than 10 patients. ${ }^{16263844}$

Three major themes were identified: (1) 'Significance of death and dying'; (2) 'Thrown in at the deep end'; and (3) 'Addressing the gap'. 

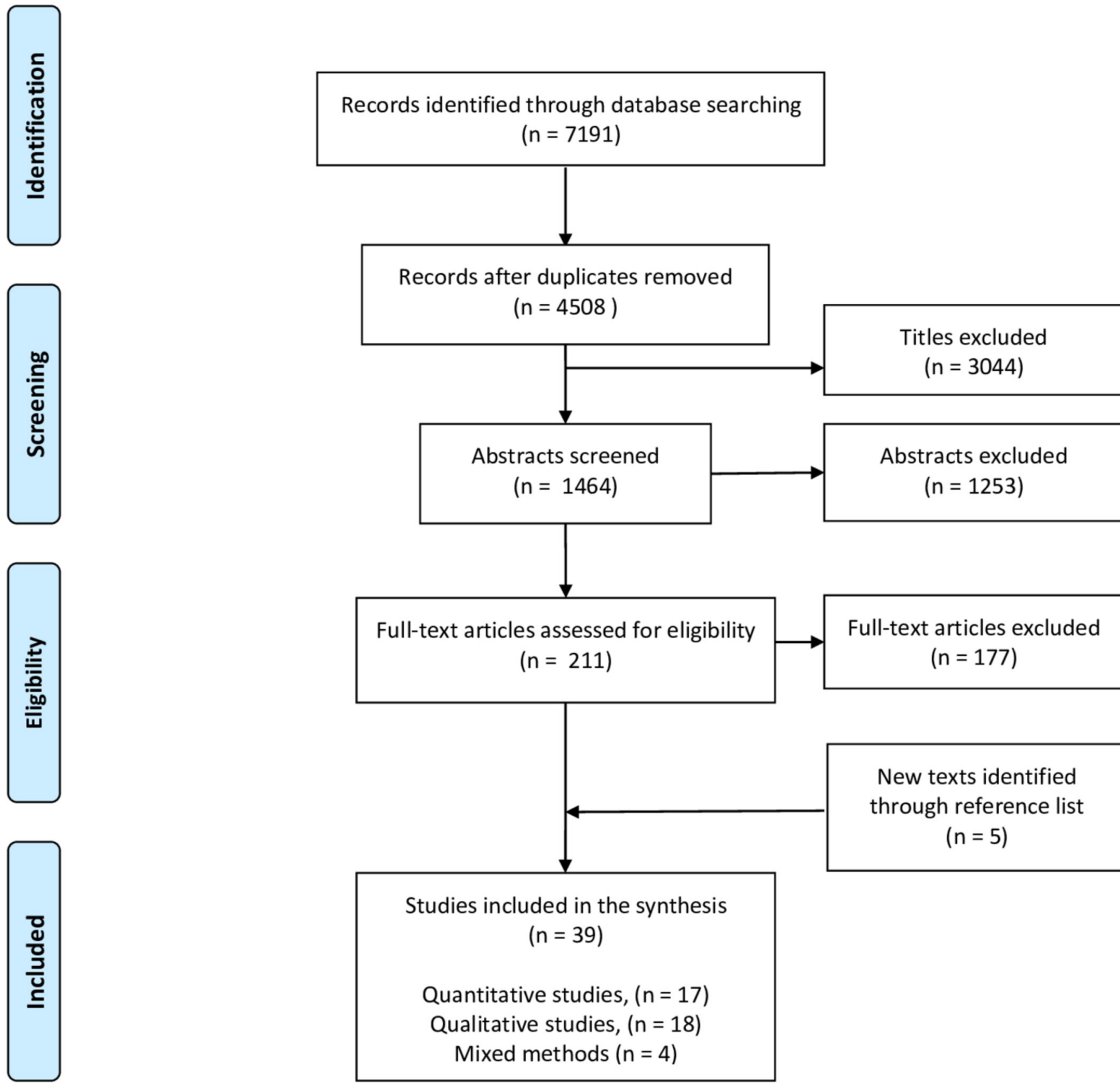

Studies included in the synthesis

$(n=39)$

Quantitative studies, $(n=17)$

Qualitative studies, $(n=18)$

Mixed methods $(n=4)$

Figure 3 PRISMA diagram. PRISMA, Preferred Reporting Items for Systematic Reviews and Meta-Analyses.

\section{Significance of death and dying}

Providing palliative and end-of-life care has a significant effect on junior doctors' emotional well-being and professional attitudes. Their first few patient deaths are memorable and powerful experiences. ${ }^{13} 1724$ 26-30 36 Junior doctors report an emotional impact, describing themselves to feel 'sad' and 'depressed', 13 22 26 '44 'anxious' and 'stressed', 1215162839 and 'guilty', 'dread', 'helplessness' and a 'sense of failure'. ${ }^{4} 264445$ Two studies revealed that $12 \%$ of junior doctors are scoring for identifiable post-traumatic stress disorder and $25 \%$ for psychological distress from caring for dying patients, as per the Post-Traumatic Stress Disorder Checklist-Civilian Version (PCL-C) scale and the 12item General Health Questionnaire (GHQ-12) measure, respectively. ${ }^{26}$ There was one exception, with a low Gough's weight of evidence study, which reported working in an inpatient hospice was not emotionally draining. ${ }^{20}$ Overall early career experiences of death and dying are significant and memorable for doctors throughout their careers. ${ }^{29} 3639$

Junior doctors' personal attitudes towards palliative and end-of-life care varied. Many report a belief in the importance of palliation, ${ }^{14} 1720212434374144454748$ some describing it as a privilege to care for patients at the end of their life. ${ }^{3045}$ Some view palliative care as similar to other aspects of medicine, describing it as problem-solving patients' symptoms. ${ }^{25} 3945$ Junior doctors describe a 'taboo hospital culture' towards patients approaching the end of their lives ${ }^{13} 30$ and a professional disengagement towards palliative 
management, ${ }^{22} 25293042$ where doctors maintain a distance from their patients 294245 and inform more junior colleagues, including students, to do the same ${ }^{1319}$ as well as prioritise other clinical tasks. ${ }^{25}$ One paper reported that junior doctors who have observed such practice subsequently questioned their careers. ${ }^{13}$

\section{Thrown in at the deep end}

Junior doctors feel unprepared and out of their depth when caring for patients approaching and at the end of their lives. $^{12-15} 182226293038404647$ Two studies, both with medium Gough's weight of evidence, had contrasting results: in one, $80 \%$ of junior doctors reported they 'felt comfortable' caring for dying patients, although only 54\% 'felt comfortable' alleviating suffering ${ }^{48}$; in another, $65 \%$ of junior doctors reported they were 'well prepared' for palliative and end-of-life care, although this was inversely correlated with their knowledge score in the area. ${ }^{49}$ Palliative and end-of-life care is more challenging for junior doctors out of hours, ${ }^{26-28} 4142$ due to frequent lack of clarity or delays in deciding about goals of care, patients being unfamiliar and having to make independent decisions.

Junior doctors commonly report being unsupported by their hospital teams in managing palliative and end-of-life care, ${ }^{13141718222627404245}$ apart from one paper in which $90 \%$ reported being able to discuss these patients with their team. ${ }^{36}$ Support is sought and received from other junior doctors as well as senior medical colleagues. ${ }^{3542}$ Senior support is variable and specialty-dependent, ${ }^{12} 1722 \quad 3842$ with most support being practical advice rather than emotional. ${ }^{17}$ Senior support is particularly lacking when senior doctors had limited experience of caring for frail older patients or greater experience in medical or surgical interventions. 37384041

Junior doctors report receiving support outside the medical team, from nursing colleagues, ${ }^{22735404147}$ palliative care specialists, ${ }^{12} 18222540$ friends and family, ${ }^{17} 39$ and online advice. ${ }^{35}$

\section{Addressing the gap}

Junior doctors perceive that palliative and endof-life care is not optimally provided in inpatient hospital settings, ${ }^{14} 27384147$ and frequently express strong requests for further training in the area. ${ }^{12} 14 \quad 15222526283234384041454648$ Junior doctors consider active participation and experiential learning as the most effective way to bridge their knowledge gap in palliative and end-of-life care. ${ }^{12} 1517$ 20-22 252931374041 Palliative care rotations, at postgraduate or undergraduate level, are seen as the best way to achieve this ${ }^{12} 1520-222528$ because the rotations or placements are valued as opportunities to address the unmet needs from medical school, which included specific and transferable skills in symptom control, professionalism, teamwork, communication, reflective practice ${ }^{172021253744}$ and in ethical-legal framework. ${ }^{17}$ There was one exception where junior doctors reported hospice placements as medically deskilling experiences, but this had a low weight of evidence. $^{20}$

Other learning methods valued by junior doctors include observation of senior colleagues 1417213137404349 and reflection in clinical contexts ${ }^{14} 17244041$ or social settings. ${ }^{1739}$ There is a preference for practical and casebased teaching 12152240414649 particularly addressing symptom control, ${ }^{15} 323840444648$ prescribing $^{21}$ the dying process ${ }^{12} 2021$ and role-playing difficult conver-

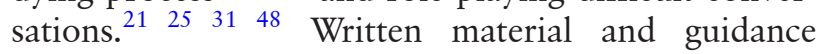
were also valued as ways to support junior doctors' prescribing $^{213340}$ and handover out of hours. ${ }^{41}$ It was reported that undergraduate and postgraduate assessments were a way to increase the curriculum priority of palliative and end-of-life care, for both educators and junior doctors.

\section{DISCUSSION}

The literature reveals that junior doctors are caring for a high number of patients needing palliative and end-of-life care. Providing such care has a significant emotional demand on them, leaving memories that persist for many years. Junior doctors feel ill-prepared and inadequately supported in this role and frequently request further training. Attitudes towards palliative and end-of-life care varied: some view it as a privilege, while others associate it with a culture of disengagement that stigmatises dying patients.

This is the first review to systematically review and synthesise the international literature concerning junior doctors' experience in providing palliative and end-of-life care, which reflects a varied weight of evidence and quality of the literature, with a range of research methods employed. It provides a timely and contemporary review of training and identifies development needs in the area, which has been rising in public interest following the Liverpool Care Pathway for the Dying Patient withdrawal and subsequent independent report. ${ }^{50}$ The report highlighted that there were multifaceted reasons for the poor care that some patients in hospitals received towards the end of their life, one of which being lack of appropriate training and support.

\section{Limitations}

We recognise that the use of the term junior doctors varies between countries, with the transition into a single-stem specialty training occurring at different time points in junior doctors' careers. The review is limited to the UK, Ireland, Canada, New Zealand and Australia, where there is greater commonality in postgraduate medical training and healthcare systems, permitting synthesis of the data. However all the themes were identified from studies in all of these countries, suggesting they are of generalisable relevance. The grey literature was not searched and 
publications were limited to the English language. The database search was augmented by reference searching to minimise the risk of relevant information being omitted; that this only identified a further five papers suggests that the database searches were robust.

\section{Interpretation of the findings}

The wider literature identifies that junior doctors worldwide feel unprepared for many areas of clinical practice, lack senior support which increases their stress in the workplace and that this experience is worse out of hours. ${ }^{51}$ The review has identified that junior doctors face these same challenges when caring for palliative and end-of-life care patients, which reflects the broader pressures junior doctors face.

However, the additional impact of palliative and end-of-life care is that there is only one chance to get it right and the significance of the associated emotional burden. Therefore greater attention is needed to junior doctors' well-being, since caring for these patients is a significant source of stress for them. ${ }^{13} 39$ This review reveals that such support is commonly not forthcoming, and instead the 'taboo culture' of a death in a hospital ${ }^{52}$ continues to prevail, with senior doctors distancing and avoiding dying patients and thus contributing to poor palliative and end-of-life care.

\section{Implications for future practice}

This review has identified a pressing need for further development of medical education in palliative and end-of-life care; this will improve the quality of patient care, equip doctors with transferrable skills, and provide them with support and resilience to deal with an emotionally demanding aspect of medicine.

The recommendations that arise are highlighted under Bloom et al's ${ }^{53}$ domains of knowledge, skills and attitudes.

\section{Knowledge}

Despite recent increases in undergraduate palliative and end-of-life care education, ${ }^{8}$ junior doctors remain frequently unprepared to care for these patients. They perceive palliative care as receiving a lower curricular priority than other aspects of medicine at both undergraduate and postgraduate levels, with specific knowledge gaps identified in symptom control, communication and the dying process. A greater focus on palliative and end-of-life care is urgently needed.

\section{Skills}

Palliative and end-of-life care is holistic and needs doctors to use a wide range of skills. Junior doctors learn and gain skills in palliative care through their personal experience and active participation. They express strong requests for further experiential learning through palliative care attachments, apprenticeship and shadowing, approaches known to improve preparedness for medical practice more generally. ${ }^{8} 51$
Attitudes

Palliative and end-of-life care places a particular emotional demand on healthcare professionals, which for some junior doctors makes palliative care unique and special, while others find it a negative emotional experience. Junior doctors need to be supported in understanding their experiences and emotional reactions when caring for the dying. Junior doctors benefit from reflection with their professional or personal colleagues; this only frequently occurs in unstructured and ad-hoc ways. Balint groups and similar structured reflection could be an effective way for junior doctors to receive emotional support, ${ }^{54}$ facilitating confidential and safe spaces to share experiences. ${ }^{19}$ The roles of senior doctors as mentors and supervisors may greatly influence their junior colleagues' attitudes towards patients who are towards the end of life.

\section{CONCLUSION}

The review has demonstrated that junior doctors frequently feel unprepared and unsupported to provide palliative and end-of-life care, with greater emotional demands than other areas of medicine. There is a pressing need for changes in undergraduate and postgraduate medical education to focus on the development of knowledge, skills and attitudes of junior doctors in this area of patient care. This can be achieved through increased curriculum content, experiential learning opportunities, and supportive and reflective practice. This will go a long way to enable future generations of junior doctors to be empowered and able to care for palliative and end-of-life care patients.

\section{Twitter Isla Kuhn@ilk21}

Contributors $\mathrm{AB}$ designed and had the concept of the work. $\mathrm{AB}$ and $\mathrm{SB}$ planned the study. $\mathrm{AB}$ and IK designed the literature search terms, with $\mathrm{AB}$ conducting the literature search from January 2000 to January 2018 and IK conducting the database search from January 2018 to August 2019. AB screened the titles, abstracts and full texts, and weighted the papers, with TM completing a sample selection at each stage. AB, TM and $\mathrm{SB}$ achieved consensus on any discrepancies in screening decisions. $\mathrm{AB}, \mathrm{SB}$ and $\mathrm{TM}$ analysed and identified the themes. $\mathrm{AB}$ wrote the draft of the article. $\mathrm{AB}, \mathrm{SB}$ and $\mathrm{BW}$ contributed to interpretation and critical revision of the article. All the authors approved the final version. $\mathrm{AB}$ is guarantor of the paper.

Funding SB is supported by the National Institute for Health Research (NIHR) Collaboration for Leadership in Applied Health Research and Care (CLAHRC) East of England at Cambridgeshire and Peterborough NHS Foundation Trust. The views expressed are those of the authors and not necessarily those of the NHS, the NIHR or the Department of Health. $\mathrm{AB}$ was supported by the Academic Foundation Programme through NIHR funding through the Health Education England East of England Deanery.

Competing interests None declared.

Patient consent for publication Not required. 
Provenance and peer review Not commissioned; externally peer reviewed.

Open access This is an open access article distributed in accordance with the Creative Commons Attribution Non Commercial (CC BY-NC 4.0) license, which permits others to distribute, remix, adapt, build upon this work noncommercially, and license their derivative works on different terms, provided the original work is properly cited, appropriate credit is given, any changes made indicated, and the use is noncommercial. See: http://creativecommons.org/licenses/by-nc/4. $0 /$.

\section{ORCID iDs}

Aamena Bharmal http://orcid.org/0000-0002-5023-3027

Stephen Barclay http://orcid.org/0000-0002-4505-7743

\section{REFERENCES}

1 Statistics OfN. National mortality statistics for England and Wales 2016, 2016.

2 Broad JB, Gott M, Kim H, et al. Where do people die? an international comparison of the percentage of deaths occurring in hospital and residential aged care settings in 45 populations, using published and available statistics. Int J Public Health 2013;58:257-67.

3 Gomes B, Higginson IJ. Where people die (1974-2030): past trends, future projections and implications for care. Palliat Med 2008;22:33-41.

4 Riley J. A strategy for end of life care in the UK. BMJ 2008;337:a943.

5 Council GM. The new doctor: recommendations on general clinical training, 2009.

6 (EAPC) EAfPC. Core competencies in palliative care: An EAPC white paper on palliative care education - Part 1. J Palliat Med 2013;20:86-91.

7 Walker S, Gibbins J, Barclay S, et al. Progress and divergence in palliative care education for medical students: a comparative survey of UK course structure, content, delivery, contact with patients and assessment of learning. Palliat Med 2016;30:834-42.

8 Lloyd-Williams M, MacLeod RDM. A systematic review of teaching and learning in palliative care within the medical undergraduate curriculum. Med Teach 2004;26:683-90.

9 Dixon-Woods M, Agarwal S, Jones D, et al. Synthesising qualitative and quantitative evidence: a review of possible methods. J Health Serv Res Policy 2005;10:45-53.

10 Gough D. Weight of evidence: a framework for the appraisal of the quality and relevance of evidence. Research Papers in Education 2007;22:213-28.

11 Barclay S, Wyatt P, Shore S, et al. Caring for the dying: how well prepared are general practitioners? A questionnaire study in Wales. Palliat Med 2003;17:27-39.

12 Bowden J, Dempsey K, Boyd K, et al. Are newly qualified doctors prepared to provide supportive and end-of-life care? A survey of Foundation year 1 doctors and consultants. J R Coll Physicians Edinb 2013;43:24-8.

13 Brennan N, Corrigan O, Allard J, et al. The transition from medical student to junior doctor: today's experiences of tomorrow's doctors. Med Educ 2010;44:449-58.

14 Centofanti J, Swinton M, Dionne J, et al. Resident reflections on end-of-life education: a mixed-methods study of the 3 wishes project. BMJ Open 2016;6:e010626.

15 Charlton R, Smith G. Perceived skills in palliative medicine of newly qualified doctors in the U.K. J Palliat Care 2000;16:27-32.

16 Clayton JM, Butow PN, Waters A, et al. Evaluation of a novel individualised communication-skills training intervention to improve doctors' confidence and skills in end-of-life communication. Palliat Med 2013;27:236-43.

17 Crawford GB, Zambrano SC. Junior doctors' views of how their undergraduate clinical electives in palliative care influenced their current practice of medicine. Acad Med 2015;90:338-44.

18 Ewing G, Farquhar M, Booth S. Delivering palliative care in an acute hospital setting: views of referrers and specialist providers. J Pain Symptom Manage 2009;38:327-40.

19 Feld J, Heyse-Moore L. An evaluation of a support group for junior doctors working in palliative medicine. Am J Hosp Palliat Care 2006;23:287-96.

20 Frearson S. Education and training: perceived educational impact, -challenges and opportunities of hospice placements for Foundation year doctors: a qualitative study. Future Healthc J 2019;6:56-60.

21 Gajebasia S, Pearce J, Redman M, et al. How can training in care of the dying be improved? Clin Teach 2019;45:12.

22 Gibbins J, McCoubrie R, Forbes K. Why are newly qualified doctors unprepared to care for patients at the end of life? Med Educ 2011;45:389-99.

23 Hayes E, Miptah HN, Beatty S, et al. In the presence of death and dying: how do new doctors feel about caring for terminally ill patients? Palliative Medicine 2016;30:NP320.

24 Hohenberg MI, Gonski P. Teach me or guide me? what do junior doctors want to learn about geriatric medicine and how, during their first year of practice? age and ageing conference: British geriatrics Society communications to the spring meeting, 2017.

25 Kawaguchi S, Mirza R, Nissim R, et al. Internal medicine residents' beliefs, attitudes, and experiences relating to palliative care: a qualitative study. Am J Hosp Palliat Care 2017;34:366-72.

26 Linane H, Connolly F, McVicker L, et al. Disturbing and distressing: a mixed methods study on the psychological impact of end of life care on junior doctors. Ir J Med Sci 2019;188:633-9.

27 Linklater GT. Educational needs of Foundation doctors caring for dying patients. J R Coll Physicians Edinb 2010;40:13-18.

28 Lloyd-Williams M. Senior house officers' experience of a six month post in a hospice. Med Educ 2002;36:45-8.

29 MacLeod RD. On reflection: doctors learning to care for people who are dying. Soc Sci Med 2001;52:1719-27.

30 Malthouse ME. Paying attention to death and dying in medical education: a narrative inquiry of junior doctors personal experiences of death and dying. Palliat Med 2012;26:602-3.

31 Mathew R, Weil A, Sleeman KE, et al. The second conversation project: -Improving training in end of life care communication among junior doctors. Future Healthc J 2019;6:129-36.

32 McCullough C, McFatter F. Prescribing at the end of life. How confident are our junior doctors? Palliat Med 2016;30:S87.

33 Mikhael J, Baker L, Downar J. Using a pocket card to improve end-of-life care on internal medicine clinical teaching units: a cluster-randomized controlled trial. J Gen Intern Med 2008;23:1222-7.

34 Minor S, Schroder C, Heyland D. Using the intensive care unit to teach end-of-life skills to rotating junior residents. Am J Surg 2009;197:814-9.

35 Miptah HN, Hayes E, Taib R, et al. Pronouncing death: how prepared do junior doctors feel to perform this role? Palliative Medicine 2016;30:NP165-6.

36 Moores TS, Castle KL, Shaw KL, et al. 'Memorable patient deaths': reactions of hospital doctors and their need for support. Med Educ 2007;41:942-6.

37 Morrison C, Forbes K. Junior doctors' learning and development in Foundation year 1 posts in palliative medicine. Palliat Med 2012;26:851-7.

38 Murray-Brown F, Curtis M, Gibbins J. Survey of Foundation year 1 doctors caring for the dying; what do they see, do and need to perform this role, following removal of the Liverpool care pathway? Palliat Med 2015;29:975-6. 
39 Paice E, Rutter H, Wetherell M, et al. Stressful incidents, stress and coping strategies in the pre-registration house officer year. Med Educ 2002;36:56-65.

40 Price S, Schofield S. How do junior doctors in the UK learn to provide end of life care: a qualitative evaluation of postgraduate education. BMC Palliat Care 2015;14:45.

41 Redman M, Pearce J, Gajebasia S, et al. Care of the dying: a qualitative exploration of Foundation year doctors' experiences. Med Educ 2017;51:1025-36.

42 Reid C, Gibbins J, Bloor S, et al. Healthcare professionals' perspectives on delivering end-of-life care within acute Hospital trusts: a qualitative study. BMJ Support Palliat Care 2015;5:490-5.

43 Robinson S, Daniels S, Jalal Q, et al. End of life care for cancer patients on a surgical ward. European Journal of Surgical Oncology 2016;42.

44 Schroder C, Heyland D, Jiang X, et al. Educating medical residents in end-of-life care: insights from a multicenter survey. J Palliat Med 2009;12:459-70.

45 Tait GR, Hodges BD. Residents learning from a narrative experience with dying patients: a qualitative study. Adv Health Sci Educ Theory Pract 2013;18:727-43.

46 Tiernan E, Kearney M, Lynch A, et al. Effectiveness of a teaching programme in pain and symptom management for junior house officers. Supportive Care in Cancer 2001;9:606-10.

47 Vivekananda-Schmidt P, Vernon B. FY1 doctors' ethicolegal challenges in their first year of clinical practice: an interview study. J Med Ethics 2014;40:277-81.

48 Weil J, Gold M, McIver S, et al. Australian resident doctors want more palliative medicine education: a survey of attitudes and perceived needs. Intern Med J 2012;42:828-30.

49 Wheatley-Price P, Massey C, Panzarella T, et al. Resident preparedness in discussing prognosis in patients with advanced lung cancer. Support Care Cancer 2010;18:491-7.

50 Do H. More care, less pathway: a review of the Liverpool care pathway, 2013.

51 Monrouxe LV, Grundy L, Mann M, et al. How prepared are UK medical graduates for practice? a rapid review of the literature 2009-2014. BMJ Open 2017;7:e13656.

52 Al-Qurainy R, Collis E, Feuer D. Dying in an acute hospital setting: the challenges and solutions. Int J Clin Pract 2009;63:508-15.

53 Bloom BS, Engelhart MD, Furst EJ, et al. Taxonomy of educational objectives, Handbook I: the cognitive domain. New York: David McKay Co Inc, 1956.

54 Van Roy K, Vanheule S, Inslegers R. Research on Balint groups: a literature review. Patient Educ Couns 2015;98:685-94. 